The final, published version of this paper appears in: Int. J. of Electronic Healthcare, Vol. 9, No. 1, pp. 60-88, 2016. doi:10.1504/IJEH.2016.078745

\title{
Process Mining in Healthcare A Systematised Literature Review
}

\begin{abstract}
Process mining is a promising approach that turns event logs into valuable insights about processes. One domain amenable to process mining is healthcare, where an enormous amount of data is generated by care processes, but where realistic care models are seldom available. In this paper, we perform a systematised literature review to assess the status of process mining, particularly in healthcare. We first provide an overview of process mining in general, and in healthcare in particular. Based on 2371 research publications related to process mining, obtained by querying six relevant search engines in May 2016, we found that the trend of publications in this domain has been growing over the past decade, especially in healthcare. Among the eleven existing literature reviews on process mining selected for further analysis, only two are systematised, and only three relate to healthcare. This paper contributes a systematised review in healthcare that is much needed to fill this void. Important challenges specific to healthcare are identified, and threats to the validity of the results are also discussed.
\end{abstract}

Keywords: Process mining, healthcare, care processes, clinical pathways, literature review

\section{Introduction}

The number of processes whose event logs are being recorded is highly increasing. Process mining is a promising approach that can use these logs and turn them into valuable insights about processes. In particular, process mining plays an important role as a bridge between traditional model-based process analysis (e.g., simulation) and data analysis techniques (e.g., data mining). This leads into a huge demand for data scientists who are not only able to analyse big data, but also to relate them to real operational processes. According to van der Aalst (2011), process mining techniques are classified into three categories: i) discovery, where a model is being created using the event logs; ii) conformance, where the data generated from the model is compared with the actual data in event logs to compare the model with reality; and iii) enhancement, where the desired data is used to improve or/and extend an existing process model.

One of the domains amenable to process mining is healthcare. In this domain, an enormous amount of data is being generated by care processes, but care models that reflect the reality are seldom available. On the other hand, healthcare expenditure is consistently rising (independently of outcomes and countries) and on average it amounts to $10 \%$ of the gross domestic product (GDP) of countries across the world (The World Bank, 2015). It is not surprising to see that the demand for high quality care at low cost is increasing, especially with our aging society. Consequently, healthcare service providers are highly motivated to use their data to improve the quality and performance of their care processes and lower their costs. Process mining is an approach that promises to support the analysis and understanding of such processes. 
Process mining is a broad area of literature that received enormous research interest in the past few years. The objective of this study is to review the literature (and trends) related to process mining and, in particular, to review surveys describing studies that have been conducted in the healthcare domain. In such context, a systematised literature review (SdLR) can help provide insight into this active area. An SdLR is less rigorous and complete than a systematic review (Kitchenham et al., 2009) and is based on the analysis of papers obtained through existing reviews rather than on the direct analysis of a large list of first-hand papers.

This paper is a SdLR that first provides a historical trend of the number of publications on process mining in general. Then, it focuses on healthcare processes and current trends. The most relevant papers reviewed in this research area are also discussed in this paper. This SdLR is more systematic and focused on healthcare than most existing reviews, and provides more accurate insight about the status of process mining in healthcare.

The organization of this paper is as follows. Section 2 presents an overview of process mining, including history, basic concepts, types, tools and techniques. Then, a typical example of application of process mining activities on an event log in healthcare is presented in Section 3. In the first step of our literature review, a brief analysis of the amount of research done in the process mining area in general is presented in Section 4. Section 5 focuses on process mining in healthcare, with its main contributions categorised and summarised. Section 6 discusses several threats to the validity of the reviews, including this one. Finally, Section 7 provides our conclusions.

\section{Overview of Process Mining}

Process mining is an emerging discipline providing novel techniques to infer valuable knowledge and information from event logs. For example, the electronic patient records in a healthcare system or the transaction logs of an enterprise resource planning system can be used to discover models describing processes, organizations, and products. Such event logs can also be used to compare reality with prior models to assess alignment (Rozinat and can der Aalst, 2008).

Process mining is positioned as a powerful tool within a broader Business Process Management (BPM) context. Process mining can also be acknowledged as a new collection of Business Intelligent (BI) techniques. Notably, most BI tools focus on some querying and reporting together with visualization techniques and are not really "intelligent" enough to convey some process mining capabilities (van der Aalst, 2011).

\subsection{Brief History of Process Mining}

According to Aldin and Cesare (2011), Cook (1996) published the first academic thesis on process mining while he was working on discovering process models from event logs in the context of software engineering. It was the first time that the term "process discovery" appeared. In 1998, Agrawal et al., from IBM Almaden Research Center, introduced process mining in the business context and called it "workflow mining". Since then, several groups focused on process mining models and developed different algorithms and implemented tools and frameworks. Particularly, van der Aalst and Weijters (2004) and their group from the Eindhoven University of Technology published the highest number of articles related to process mining. 


\subsection{Three Types of Process Mining}

In general, process mining activities are categorised into three main types: i) Discovery, ii) Conformance, and iii) Enhancement (see Figure 1).

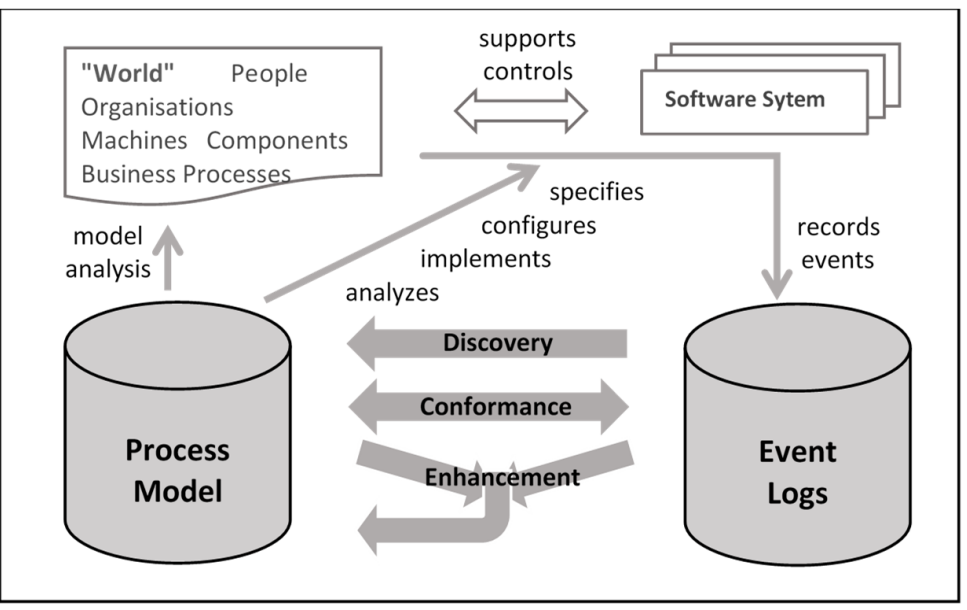

Figure 1. Three types of process mining: (1) Discovery, (2) Conformance, and (3) Enhancement (inspired from van der Aalst, 2011)

- Discovery: Here, the aim is to produce a process model, e.g., a Petri net (ISO/IEC, 2004) or a BPMN model (OMG, 2011), using event logs. There is no prior model involved in a process discovery technique. The inferred model should be able to describe the observed behaviour of a process. The $\alpha$ algorithm (Li et al., 2007), which is able to discover a model based on sequences of events, is an example of discovery technique. Note that some models describing the data or organizational perspectives may be discovered (van der Aalst et al., 2014; van der Aalst, 2011). Process discovery is acknowledged as the most prominent process mining technique.

- Conformance: In this type, an existing process model is compared with observed behaviour in the event logs (Rozinat and van der Aalst, 2008). Deviations between the model and the logs (e.g., activities in the model that do not exist in the log or vice versa) can be further analysed. Conformance checking can be used for business auditing and compliance checking. For example, replaying the event logs on top of the process model can highlight undesirable deviations and suggests fraud or inefficiencies. Conformance checking techniques can also be used for measuring the performance of process discovery algorithms and for repairing unaligned models.

- Enhancement: Whereas conformance checking assesses the alignment between reality and model, the third type of process mining aims to change or improve the a priori model. Note that it is supposed that a model, either discovered or produced manually, already exists (van der Aalst, 2011; 2012). There are two types of enhancement activities: repair, where the model is modified to reflect reality better and bring the model closer to the reality, and 
extension, where a new perspective (time, costs, risks, resource usage, etc.) is added to the process model by cross-correlating it with the event logs. For example, one can identify bottlenecks by replaying timestamps in the event logs of a process model.

Figure 2 shows the inputs and outputs of the three types of process mining.

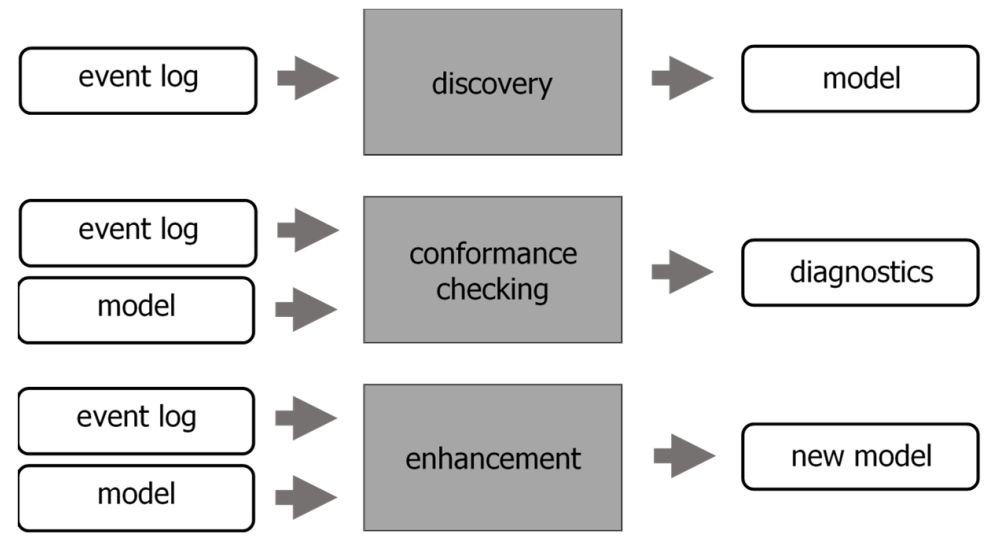

Figure 2. Three Basic Types of Process Mining, with Inputs and Outputs (Inspired from van der Aalst et al., 2012)

\subsection{Four Main Quality Dimensions of a Model}

It is challenging to determine the quality of a process model discovered through a process mining algorithm. There are many dimensions that could be considered for such models. Four main quality dimensions are defined by van der Aalst (2011) to assess the quality of a model: fitness, simplicity, precision, and generalization.

- Fitness: A model is perfect in terms of fitness if all traces in the logs can be produced by the model from beginning to end.

- Complexity: This factor is defined by the number of nodes and edges used in the graph of the model.

- Precision: This dimension is good if the model is not "under-fitting". If the model allows for many other behaviours, even if there are no signs in the current logs that imply this behaviour, the model is under fitting and is not precise enough.

- Generalization: This factor is related to avoiding "overfitting". A model is not general enough if it explains the specific sample log, but another sample $\log$ of the same process produces a completely dissimilar process model.

Process mining algorithms should manage the trade-offs between these four main dimensions.

\subsection{Process Discovery Algorithms}

There are many process mining algorithms proposed by researchers and practitioners. Each of these algorithms has different performances for different kinds of processes. As a result, 
it is difficult to select an appropriate process mining algorithm for a given application domain (Wang et al., 2013). There are four typical characteristics of process discovery algorithms, with limitations further discussed by van der Aalst (2011):

- Representational bias: Most algorithms are not necessarily able to find all processes. There are some circumstances in event logs that cannot be covered by some algorithms. Some typical representational limitations forced by some process discovery algorithms are inabilities to represent: concurrency, (arbitrary) loops, silent actions, duplicate actions, non-free-choice behaviour, OR-splits/joins, and hierarchy.

- Ability to deal with noise: Noisy behaviour, i.e., infrequent or exceptional behaviour, should not be considered in the discovered model, as users typically need to see the mainstream behaviour. Yet, it is hard to extract meaningful knowledge from activities or patterns that are extremely infrequent. Mature algorithms deal with this issue by abstracting from infrequent behaviour.

- Completeness notion assumed: Some algorithms work with the assumption that the event log includes all possible traces, i.e., a strong completeness assumption. This is not realistic and results in overfitting models. However, a weak completeness assumption tends to result in under-fitting models.

- Approach used: Four typical families of approaches are: direct algorithmic (when the model is directly constructed from the event log), two-phase (when a "low-level model" is made first and then this model is converted into a "high-level model" that can show concurrency and more advanced patterns), computational intelligence (evolutionary approaches in which the model is constructed iteratively), and partial (similar to the discovery of association rules, but with respect to the order of events).

Some of the main process discovery algorithms include the following (van der Aalst, 2011):

- $\quad \boldsymbol{\alpha}$-algorithm: This algorithm shows many of the general ideas used by many process mining algorithms and aids to understand the concept of process discovery (Li et al., 2007). This algorithm takes an event log as input and makes a Petri net showing the behaviour detailed in the log, including concurrency. However, the $\alpha$-algorithm is not a very practical mining technique as it has some problems with noise, infrequent behaviour, incomplete behaviour, and complex routing constructs.

- Heuristic Mining: Heuristic mining algorithms, described by Weijters and Ribeiro (2011), use graphs whose nodes represent activities and arcs represent causal dependencies. These algorithms consider frequencies of events and sequences when making a process model. The basic idea is that infrequent paths should not be accommodated into the model.

- Genetic Process Mining: This is an example of a process discovery algorithm that adopts a computational intelligence approach (de Medeiros et al., 2007). Like in any genetic algorithm, there are four main steps in this algorithm: initialization, selection, reproduction, and termination.

- Region-Based Mining: After converting an event log into a low-level transition system, one can synthesise a Petri net from it. In turn, this Petri net can be used to make a process model in terms of some other high-level 
notation (e.g., BPMN or UML activity diagrams). Folding a large transition system into a smaller Petri net by detecting concurrency is the challenge here. The main idea is to find regions that correspond to places. A region is a set of states such that all activities in the transition system "agree" on the region (Bergenthum et al., 2007).

Rozinat et al. (2007) proposed an evaluation framework to compare the performance of algorithms, and to evaluate the validity of their results.

\subsection{Process Mining Tools}

van Dongen et al. (2005), from the Eindhoven University of Technology, developed a "pluggable" environment for process mining: the ProM framework (Process Mining Group, 2010). This framework is flexible in terms of the input and output formats, and allows reusing code throughout the implementation of novel process mining ideas. ProM uses MXML, SA-MXML (de Medeiros et al., 2008), or XES (2016) as input format. Some import tools such as ProMimport and XESame are able to convert data from several sources into event logs. The ProM framework is well-known among researchers and is the most popular and frequently utilised framework amongst process mining practitioners (Claes and Poels, 2013).

Process mining capabilities are also provided by a growing number of commercial analysis tools such as Disco (Fluxicon, 2016), ARIS Process Performance Manager (Software AG, 2012), Celonis Process Mining (Celonis, 2016), ProcessAnalyzer (QPR, 2015), Perceptive Process Mining (Lexmark International, 2016), and Interstage Process Discovery (Fujitsu, 2016). These commercial tools embed many of the ideas developed in the context of ProM.

\subsection{Process Mining Manifesto}

In 2012, the IEEE Task Force on Process Mining released the first version of the Process Mining Manifesto (van der Aalst et al., 2012). Fifty-three organizations and seventy-seven process mining experts supported and contributed to this manifesto. In this document, six guiding principles are defined to prevent users/analysts from making mistakes when applying process mining in real situations. Processes and information should be aligned in order to meet requirements related to compliance, efficiency, and customer service. In the manifesto, eleven important challenges are explained.

\section{Process Mining in Healthcare: An Example}

As discussed in Section 1, one area that is amenable to process mining is healthcare. Healthcare Information Systems (HIS) have numerous tables consisting of patient-related event data. Such data can be exploited to improve care processes while reducing costs (Mans et al., 2015). Process mining techniques can play an important role in this regards, e.g., to improve compliance and performance. In this section, we show an example of process mining in healthcare.

To be able to improve processes, it is essential to understand what is really happening (process discovery) and analyse and interpret deviations from the expected process model (conformance checking). Furthermore, adding some perspective (times, costs, risks, 
resource usage, etc.) on top of the process model can help us identify and diagnose the roots of inefficiencies such as bottlenecks (enhancement).

For example, Table 1 shows event log of activities of 15 patients who have used the service of a health care system, namely the screening and diagnosis of gestational diabetes. Note that this table is not necessarily an original table that is stored in a database. It may be prepared from some raw tables that recorded all activities in order of the time that each event (activity) has happened.

Table 1. Event Log Example for Gestational Diabetes

\begin{tabular}{|c|c|c|}
\hline Case & Activities & Encoding \\
\hline Patient1 & $<$ admission, regular test, check the result, send the result $>$ & $<\mathrm{a}, \mathrm{b}, \mathrm{c}, \mathrm{g}\rangle$ \\
\hline Patient2 & $\begin{array}{l}<\text { admission, regular test, check the result, request for advanced } \\
\text { test, advanced test, check the result, send the result }>\end{array}$ & $\begin{array}{l}<a, b, c, d, e \\
\quad c, g>\end{array}$ \\
\hline Patient3 & $\begin{array}{l}<\text { admission, regular test, check the result, request for repetition, } \\
\text { regular test, check the result, send the result }>\end{array}$ & $\begin{array}{l}<a, b, c, f, b \\
\quad c, g>\end{array}$ \\
\hline Patient4 & $\begin{array}{l}<\text { admission, regular test, check the result, request for repetition, } \\
\text { regular test, check the result, request for advanced test, advanced } \\
\text { test, check the result, send the result }>\end{array}$ & $\begin{array}{l}<a, b, c, f, b \\
c, d, e, c, g>\end{array}$ \\
\hline Patient5 & $\begin{array}{l}<\text { admission, regular test, check the result, request for advanced } \\
\text { test, advanced test, check the result, request for advanced test, } \\
\text { advanced test, check the result, request for advanced test, } \\
\text { advanced test, check the result, send the result }>\end{array}$ & $\begin{array}{l}<a, b, c, d, e \\
\text { c, d,e,c, d } \\
\quad \text { e, c, g> }\end{array}$ \\
\hline Patient6 & $\begin{array}{l}<\text { admission, regular test, check the result, request for advanced } \\
\text { test, advanced test, check the result, send the result }>\end{array}$ & $\begin{array}{l}<a, b, c, d, e, \\
\quad c, g>\end{array}$ \\
\hline Patient7 & $\begin{array}{l}\text { <admission, regular test, check the result, request for advanced } \\
\text { test, advanced test, check the result, send the result }>\end{array}$ & $\begin{array}{l}<a, b, c, d, e, \\
\quad c, g>\end{array}$ \\
\hline Patient8 & $<$ admission, regular test, check the result, send the result $>$ & $<\mathrm{a}, \mathrm{b}, \mathrm{c}, \mathrm{g}>$ \\
\hline Patient9 & $\begin{array}{l}<\text { admission, regular test, check the result, request for repetition, } \\
\text { regular test, check the result, request for advanced test, advanced } \\
\text { test, check the result, send the result }>\end{array}$ & $\begin{array}{l}<a, b, c, f, b \\
c, d, e, c, g>\end{array}$ \\
\hline Patient 10 & $\begin{array}{l}<\text { admission, regular test, check the result, request for advanced } \\
\text { test, advanced test, check the result, send the result }>\end{array}$ & $\begin{array}{l}<a, b, c, d, e \\
\quad c, g>\end{array}$ \\
\hline Patient 11 & $\begin{array}{l}<\text { admission, regular test, check the result, request for repetition, } \\
\text { regular test, check the result, send the result }>\end{array}$ & $\begin{array}{l}<a, b, c, f, b \\
\quad c, g>\end{array}$ \\
\hline Patient12 & $<$ admission, regular test, check the result, send the result $>$ & $<a, b, c, g>$ \\
\hline Patient13 & $\begin{array}{l}<\text { admission, regular test, check the result, request for advanced } \\
\text { test, advanced test, check the result, send the result }>\end{array}$ & $\begin{array}{l}<a, b, c, d, e \\
\quad c, g>\end{array}$ \\
\hline Patient14 & $<$ admission, regular test, check the result, send the result $>$ & $<a, b, c, g\rangle$ \\
\hline Patient 15 & $<$ admission, regular test, check the result, send the result $>$ & $<a, b, c, g>$ \\
\hline
\end{tabular}

If we use short names to encode the activities (e.g., $a=$ admission), we obtain the traces shown in the third column of Table 1 . The attributes of the various events, e.g., timestamp, resource, and data, are not shown in the table. There are some activities that are repeated for more than one patient; if we aggregate repeated ones, the event log can be shown as follows:

$$
\begin{aligned}
\mathrm{L}= & {\left[<a, b, c, g>^{5},<a, b, c, d, e, c, g>^{5},<a, b, c, f, b, c, g>^{2},\right.} \\
& \left.<a, b, c, f, b, c, d, e, c, g>^{2},<a, b, c, d, e, c, d, e, c, d, e, c, g>^{1}\right]
\end{aligned}
$$


After analysing the events logs and the activities executed for patients, a process model such as the one shown in Figure 3 can be discovered. In this example, we use the $\alpha$ algorithm (Li et al., 2007) to obtain the control-flow model from event logs. This model is able to reproduce the traces observed and shown in Table 1.

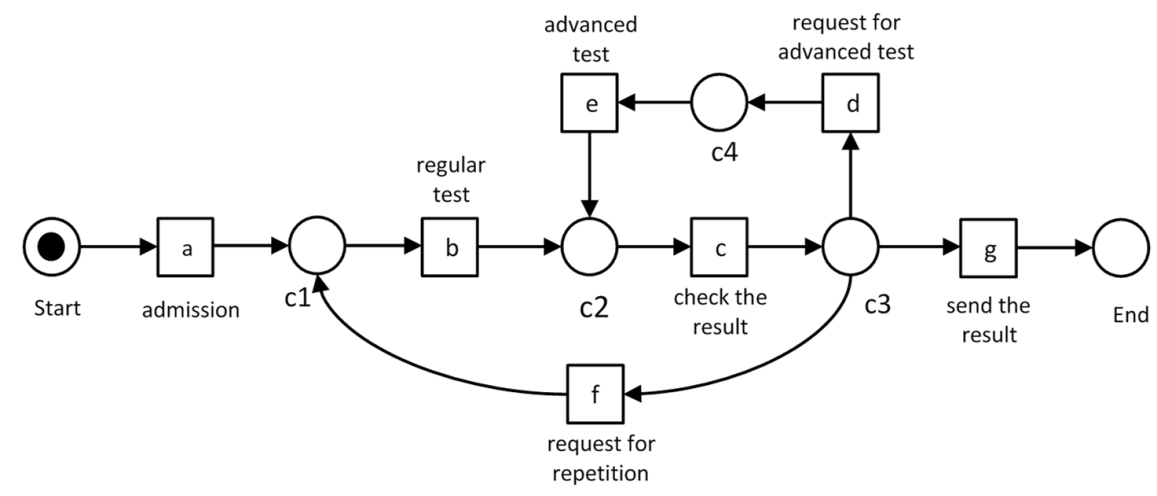

Figure 3. Process Model Discovered from an Event Log, Using the $\alpha$-Algorithm

The control-flow is represented in terms of a Petri net, where transitions (squares) represent activities and places (circles) represent states. Transitions are connected through places. A transition is enabled and its corresponding activity can execute, if all input places hold a token (black dot). A token here essentially represents a patient.

The process of screening and diagnosis of gestational diabetes starts by the admission of a pregnant patient. Transition admission has just one input place (start) and this place primarily has a token representing a patient. Hence, the corresponding activity is enabled and can happen. This is also called firing. When firing, the transition uses one token from each of its input places and produces one token for each of its output places. Hence, the firing of transition admission removes the token from input place start and produces a token for output place c1. Figure 3 shows the initial state (also called as marking), which consists of one token in place start. The marking after firing transition admission has a tokens in place c2. This goes on until the token gets to 33 , where there is a choice between three activities, and possible iterations through other activities. The process ends with a token in place end.

There may be other information such as the resource (physician or nurse) executing the activity, the time of the events, or data that characterise the patient. By replaying the event $\log$ on top of the model shown in Figure 3, we can learn additional perspectives and enrich the model, as suggested in Figure 4 (some kind of conformance and enhancement).

As Figure 4 shows, according to Mans et al. (2015), one can extend the process model with additional perspectives: the organizational perspective ("What are the roles and which resources are doing particular activities?"), the case perspective ("Which characteristics of a case impact on a particular decision?"), and the time perspective ("Where are the bottlenecks in the process?"). 


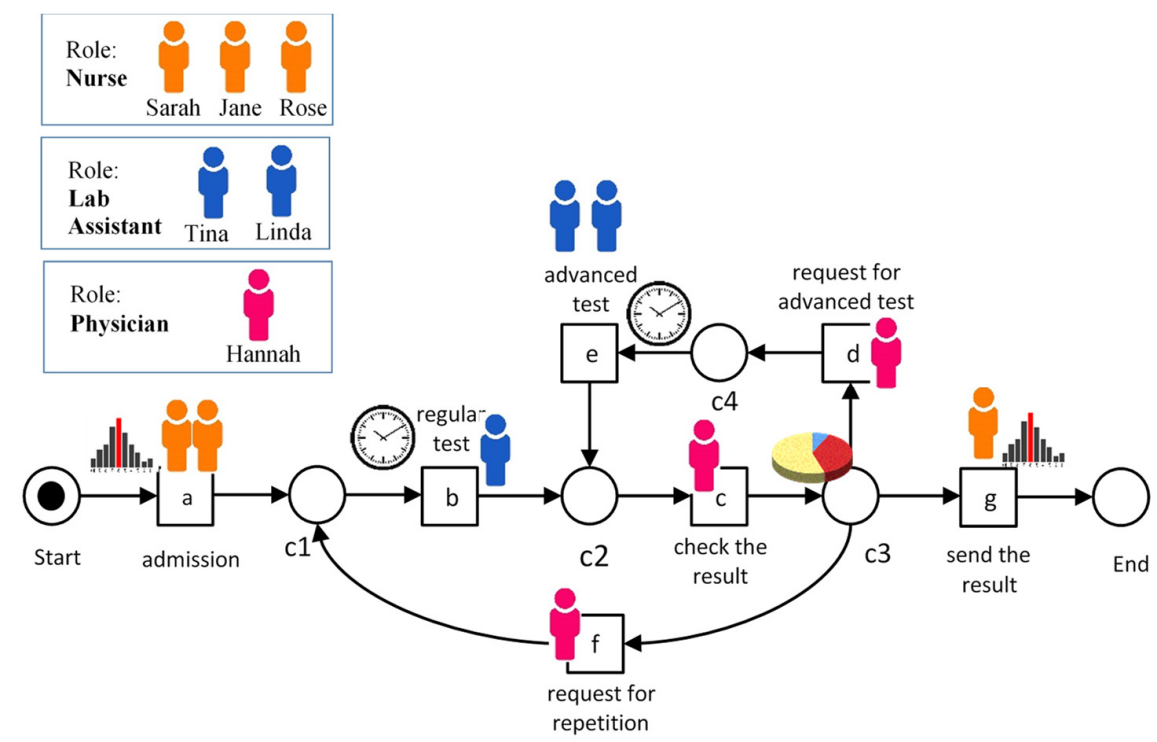

Figure 4. Process Mining is Also Used to Understand Deviations, Analyse Bottlenecks, and Monitor Organizational Behaviour

Analysis of the event log may disclose that Hannah is the only employee that performs the activities check the result, request for advanced test and request for repetition. This advises that there is a "physician role" and that only Hannah has this role. Activities regular test and advanced test are performed only by Tina and Linda, suggesting some "Lab Assistant" related to this activity, etc. Techniques for organizational process mining (van der Aalst, 2011) will learn such organizational structures and relate activities to resources through roles. By taking advantage of resource information in the $\log$, the organizational perspective can be added to the process model. Similarly, information on timestamps and frequencies can be used for adding performance related information to the model. Figure 4 shows that we can measure the time that passes between a request for advanced test (activity d) and the actual test (activity e). If this time is unusually long, process mining can identify the problem and discover possible root causes. If the event log has case-related information, this can be used to analyse the decision-making activities in the process. For instance, through this analysis, it may be learned that older patients require multiple operations (Mans et al., 2015).

\section{Literature Review of Process Mining in Healthcare}

In this section, we aim to review the papers related to process mining in healthcare. The trends of research on process mining in general and in healthcare are discussed. Important literature-review papers generally related to process mining are first briefly reviewed. Then, we narrow down the scope and focus on the healthcare domain.

Inspired by the work of Kitchenham et al. (2009), we did our systematised literature review to address the following three research questions: 
RQ1. What is the trend of volume of research activities related to process mining and what is the share of healthcare in this trend?

RQ2. Are there any literature reviews specifically related to process mining? If so, how many of them are done in a systematic way and what are their main contributions? Is any of them related to care processes in particular?

RQ3. Do the literature reviews that are published give us a good understanding about process mining, especially in healthcare?

In this research, we considered four of the most relevant search engines in information technologies, namely Scopus, Google Scholar, IEEE Explore, and Academic Search Complete (ASC). In addition, we also considered PubMed (also covering Medline), Embase, Clinical Evidence, and the Cochrane Library, which are four healthcare indexes. The last four ones were included to cover the papers that are likely included only in healthcare databases. To conserve the ability of trend analysis, we consciously did not limit our review to a particular time period.

\subsection{Trend of Volume of Research Activities}

First, we ran an abstract query looking for papers related to process mining in general. We searched for "process mining" within the text of papers and, as shown in the first row of Table 2, Google Scholar and Scopus returned thousands, IEEE Explore returned about one thousand, ASC less than three hundreds, and PubMed and Embase returned less than 30 papers each. Two other search engines, Clinical Evidence and Cochrane Library, did not return any paper relevant to process mining. Based on these numbers, for the first four engines, we limited the search to metadata (title, abstract and keywords) in order to find manageable, meaningful and relevant results. As Google Scholar is not able to search within abstracts and keywords, it was limited to only the title (patents and citations were also excluded). This limitation reduced the number of results dramatically and enabled us to analysis the trend of research activities in process mining while avoiding noise from less relevant papers. However, if one wants to sensitise the papers and review the results in details, the query needs to be further constrained. The result of the second query is shown in the second row of Table 2.

Table 2. Number of Papers Returned by the Search Engines

\begin{tabular}{|c|l|c|c|c|c|c|c|}
\hline & \multicolumn{1}{|c|}{ Search within } & Scopus & $\begin{array}{c}\text { Google } \\
\text { Scholar }\end{array}$ & $\begin{array}{c}\text { IEEE } \\
\text { Explore }\end{array}$ & ASC & PubMed & Embase \\
\hline 1 & Full text & 4,177 & 10,200 & 1,009 & 248 & 29 & 23 \\
\hline 2 & Limited & 1,845 & 1,120 & 343 & 139 & 29 & 23 \\
\hline 3 & Extra limited & 1,780 & 11,20 & 343 & 139 & 29 & 23 \\
\hline 4 & $\begin{array}{l}\text { Removing duplications, } \\
\text { independently within } \\
\text { each search engine }\end{array}$ & 1,744 & 1,011 & 342 & 139 & 28 & 21 \\
\hline 5 & $\begin{array}{l}\text { Removing duplications, } \\
\text { across all engines }\end{array}$ & \multicolumn{7}{|c|}{$\mathbf{2 , 3 7 1}$} \\
\hline
\end{tabular}

Reviewing the returned papers published before 2001 revealed that they are mostly related to processes in the chemical or mining industry. So, we added an extra constraint to the second query to exclude them: "process mining" AND NOT ("mineral" OR "caving" OR "chemistry" OR "chemical"), with impact only on Scopus; Google 
Scholar only searches within the titles, and the other engines already focus on domains other than the mineral industry.

Then, we focused on the results to find and eliminate duplicated papers in each search engine, in a trackable manner. The fourth row of Table 2 shows the numbers of papers after removing this kind of duplication. Finally, we searched for duplicates across the results of the six search engines and after removing them, 2371 papers remained. In order to gain insight about the contribution of each search engine in finding the papers related to process mining, Table 3 shows all combinations of the search engines together with the number of papers returned by each combination. For example, row 1 shows that 1043 papers out of 2371 unique papers (43.99\%) have been found only by Scopus (and the other 5 search engines have not been able to return them). Row 2 of Table 3 shows that 335 papers have been found commonly by Scopus and Google Scholar but not by the other four search engines. Logically, 63 combinations could be generated from 6 search engines. However, Table 3 only cites the 28 combinations for which there are actually papers returned.

Table 3. Contribution of search engines in finding 2,371 unique papers related to process mining

\begin{tabular}{|c|c|c|c|c|c|c|c|c|}
\hline Search engine: & Scopus & $\begin{array}{c}\text { Google } \\
\text { Scholar }\end{array}$ & $\begin{array}{c}\text { IEEE } \\
\text { explore }\end{array}$ & ASC & PubMed & Embase & \multirow[b]{2}{*}{ Number } & \multirow[b]{2}{*}{ Portion } \\
\hline $\begin{array}{c}\text { Papers } \\
\text { returned: } \\
\text { (Percent) }\end{array}$ & $\begin{array}{c}1742 \\
(73.5 \%)\end{array}$ & $\begin{array}{c}1011 \\
(42.6 \%)\end{array}$ & $\begin{array}{c}342 \\
(14.4 \%)\end{array}$ & $\begin{array}{c}140 \\
(5.9 \%)\end{array}$ & $\begin{array}{c}28 \\
(1.2 \%)\end{array}$ & $\begin{array}{c}21 \\
(0.9 \%)\end{array}$ & & \\
\hline 1 & $\checkmark$ & $x$ & $x$ & $x$ & $x$ & $x$ & 1043 & $43.99 \%$ \\
\hline 2 & $\checkmark$ & $\checkmark$ & $x$ & $x$ & $x$ & $x$ & 335 & $14.13 \%$ \\
\hline 3 & $\checkmark$ & $x$ & $\checkmark$ & $x$ & $x$ & $x$ & 161 & $6.79 \%$ \\
\hline 4 & $\checkmark$ & $x$ & $x$ & $\checkmark$ & $x$ & $x$ & 39 & $1.64 \%$ \\
\hline 5 & $\checkmark$ & $\checkmark$ & $\checkmark$ & $x$ & $x$ & $x$ & 108 & $4.56 \%$ \\
\hline 6 & $\checkmark$ & $\checkmark$ & $x$ & $\checkmark$ & $x$ & $x$ & 26 & $1.10 \%$ \\
\hline 7 & $\checkmark$ & $\checkmark$ & $x$ & $x$ & $\checkmark$ & $x$ & 5 & $0.21 \%$ \\
\hline 8 & $\checkmark$ & $x$ & $\checkmark$ & $\checkmark$ & $x$ & $x$ & 3 & $0.13 \%$ \\
\hline 9 & $\checkmark$ & $x$ & $x$ & $x$ & $\checkmark$ & $\checkmark$ & 4 & $0.17 \%$ \\
\hline 10 & $\checkmark$ & $\checkmark$ & $\checkmark$ & $\checkmark$ & $x$ & $x$ & 3 & $0.13 \%$ \\
\hline 11 & $\checkmark$ & $\checkmark$ & $\checkmark$ & $x$ & $\checkmark$ & $x$ & 1 & $0.04 \%$ \\
\hline 12 & $\checkmark$ & $\checkmark$ & $x$ & $\checkmark$ & $\checkmark$ & $x$ & 2 & $0.08 \%$ \\
\hline 13 & $\checkmark$ & $\checkmark$ & $x$ & $x$ & $\checkmark$ & $\checkmark$ & 4 & $0.17 \%$ \\
\hline 14 & $\checkmark$ & $x$ & $\checkmark$ & $x$ & $\checkmark$ & $\checkmark$ & 1 & $0.04 \%$ \\
\hline 15 & $\checkmark$ & $x$ & $x$ & $\checkmark$ & $\checkmark$ & $\checkmark$ & 4 & $0.17 \%$ \\
\hline 16 & $\checkmark$ & $\checkmark$ & $\checkmark$ & $\checkmark$ & $\checkmark$ & $x$ & 1 & $0.04 \%$ \\
\hline 17 & $\checkmark$ & $\checkmark$ & $\checkmark$ & $x$ & $\checkmark$ & $\checkmark$ & 1 & $0.04 \%$ \\
\hline 18 & $\checkmark$ & $\checkmark$ & $x$ & $\checkmark$ & $\checkmark$ & $\checkmark$ & 1 & $0.04 \%$ \\
\hline 19 & $x$ & $\checkmark$ & $x$ & $x$ & $x$ & $x$ & 497 & $20.96 \%$ \\
\hline 20 & $x$ & $\checkmark$ & $\checkmark$ & $x$ & $x$ & $x$ & 21 & $0.89 \%$ \\
\hline 21 & $x$ & $\checkmark$ & $x$ & $\checkmark$ & $x$ & $x$ & 4 & $0.17 \%$ \\
\hline 22 & $x$ & $\checkmark$ & $x$ & $x$ & $\checkmark$ & $x$ & 2 & $0.08 \%$ \\
\hline 23 & $x$ & $x$ & $\checkmark$ & $x$ & $x$ & $x$ & 41 & $1.73 \%$ \\
\hline 24 & $x$ & $x$ & $\checkmark$ & $\checkmark$ & $x$ & $x$ & 1 & $0.04 \%$ \\
\hline 25 & $x$ & $x$ & $x$ & $\checkmark$ & $x$ & $x$ & 56 & $2.36 \%$ \\
\hline 26 & $x$ & $x$ & $x$ & $x$ & $\checkmark$ & $x$ & 1 & $0.04 \%$ \\
\hline 27 & $x$ & $x$ & $x$ & $x$ & $\checkmark$ & $\checkmark$ & 1 & $0.04 \%$ \\
\hline 28 & $x$ & $x$ & $x$ & $x$ & $x$ & $\checkmark$ & 5 & $0.21 \%$ \\
\hline & & & & & To & & 2371 & $100 \%$ \\
\hline
\end{tabular}




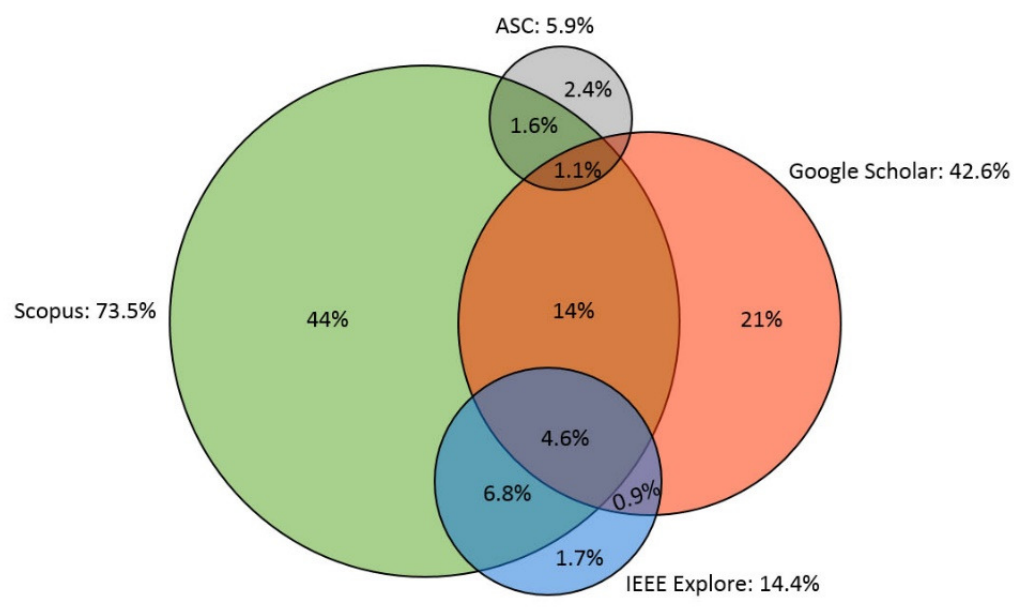

Figure 5. Percentages of Papers Returned by Four Search Engines, with Duplicates as Set Intersections

As Table 3 shows, some combinations have a very small number of papers. In order to further visualize some of this information, we consider only the rows that have resulted in more than five papers. These rows cover $99.98 \%$ of all papers and are highlighted in Table 3. Based on these rows, Figure 5 illustrates the distribution of unique papers between the search engines' output (to scale). Considering Figure 5, only 4.1 percent of the papers have been covered neither by Scopus nor by Google Scholar. Thus, searching with these two search engines results in the vast majority of papers in the process mining domain, although not all of them. Moreover, if we only use one of these two search engines, we will miss a significant number of relevant papers. Also, the last three rows of Table 3 show the contribution of PubMed and Embase in finding the papers. Row 26 shows that only one paper (out of 2371 papers) is only returned by PubMed. Row 28 shows that the number of papers found only by Embase is 5 (out of 2371 papers). According to these results, if we ignore PubMed and Embase, we only miss 7 papers ( $0.3 \%$ of all papers).

Based on the year of publication, the trend of the number of unique publications related to process mining is illustrated in Figure 6. According to this figure, process mining is a growing research topic that has emerged in the last decade, and recently it has received considerably higher research interest. 


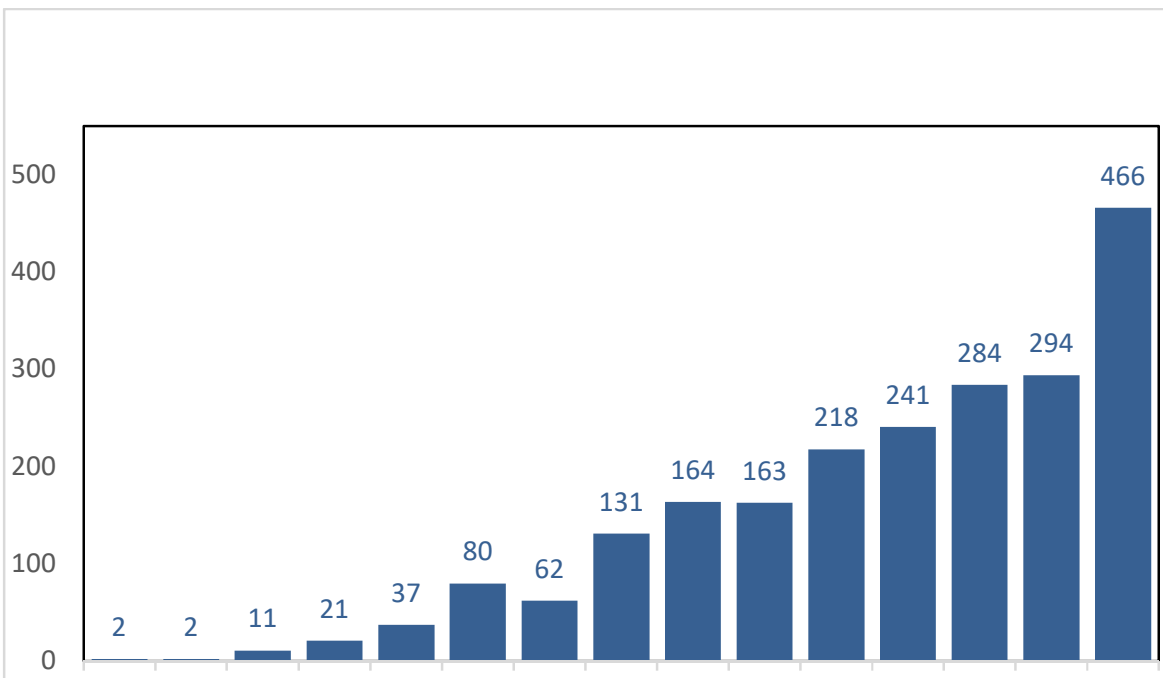

200120022003200420052006200720082009201020112012201320142015

Figure 6. Number of Process-Mining-Related Publications, Per Year

In order to find the share of the healthcare domain in process mining related research, we defined another query with additional constraints. This time, our query was "process mining" AND (healthcare OR clinic OR hospital OR care OR clinical OR health). This query covered the essential keywords in the healthcare domain. The raw results of the search engines, where the query was limited to titles and keywords, are shown in Table 4. Given the specialised area of PubMed and Embase, it is not surprising to see that a majority of papers returned by these databases are related to healthcare.

Table 4. Process Mining in Healthcare - Automatic Search Results

\begin{tabular}{|c|c|c|c|c|c|c|}
\hline & Scopus & $\begin{array}{c}\text { Google } \\
\text { Scholar }\end{array}$ & $\begin{array}{c}\text { IEEE } \\
\text { Explorer }\end{array}$ & ASC & PubMed & Embase \\
\hline $\begin{array}{c}\text { Search } \\
\text { Results }\end{array}$ & 128 & 58 & 34 & 19 & 23 & 16 \\
\hline
\end{tabular}

By comparing the 278 papers that the search engines have returned and by removing their duplicates, we get a total of 168 unique papers. A quick assessment of these papers confirmed that our customised queries have worked well as a majority of these papers are relevant.

To complete the answer to research question RQ1, Figure 7 illustrates the number of publications related to process mining in healthcare and its growing trend. Moreover, the share of research papers related to healthcare in the whole process mining domain is shown in Figure 8. It can be seen from this latter figure that this share has a fairly increasing trend over the recent five years. 


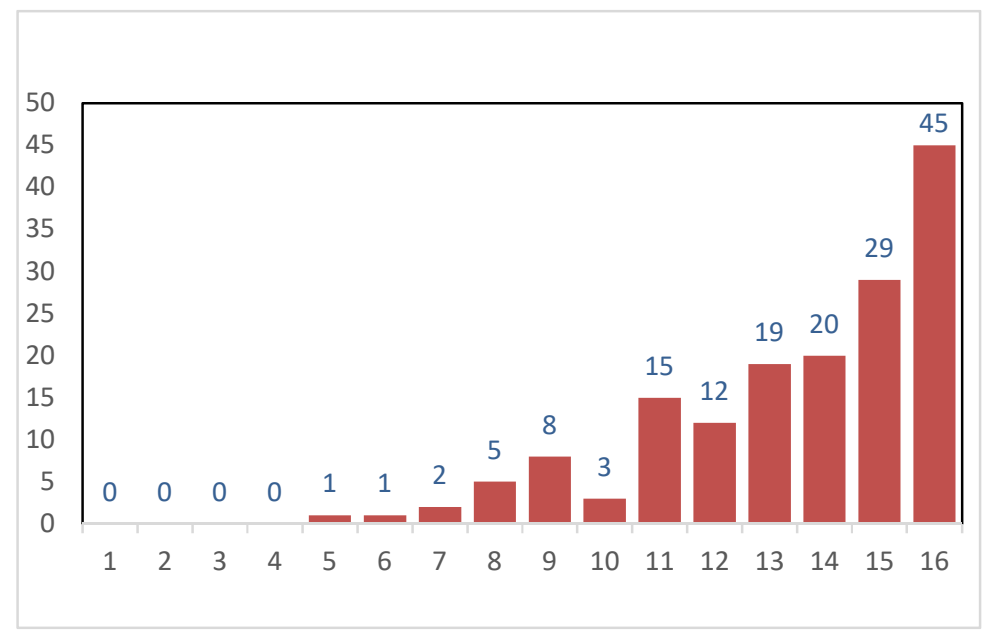

Figure 7. Number of Publications Related to Process Mining in Healthcare, Per Year

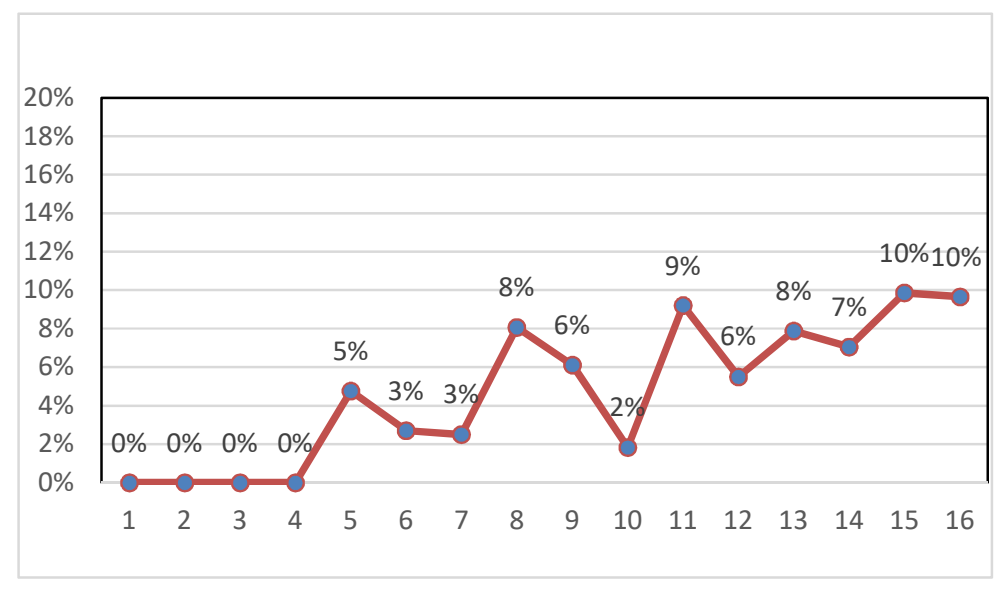

Figure 8. Share of Healthcare in Process Mining Publications, Per Year

\subsection{Looking for Existing Literature Reviews}

In order to answer research question RQ2, we adopted a two-step method. In the first step, we looked for papers that did a literature review on process mining, regardless of any specific domain. Then, in the second step, we selected the papers that are related to the healthcare domain. This method not only answers our question, but also provides us with insight about the endeavours targeting literature reviews on process mining.

Our query was "process mining" AND (survey OR literature OR review) and resulted in 37 papers. We excluded papers that: 
- Only cited process mining or its derivatives to acknowledge its existence or to mention it in some example.

- Clearly were related to process mining but were not clearly reviews.

- Were not clearly related to neither process mining nor literature reviews.

Our selection led to eleven papers that reported a literature review of process mining (Table 5). In the following section, we will review these eleven specific papers.

Table 5. Selected Literature Reviews on Process Mining, with Citations from Google Scholar on May 13, 2016

\begin{tabular}{|c|c|c|c|c|c|}
\hline Paper & Year & Focus & Systematic? & Source & Citations \\
\hline Agarwal \& Singh & 2014 & \multirow{2}{*}{ Tools } & No & Journal & 0 \\
\hline Claes \& Poels & 2013 & & No & Conference & 16 \\
\hline Tiwari et al. & 2008 & \multirow{6}{*}{$\begin{array}{l}\text { Algorithms } \\
\text { and issues }\end{array}$} & No & Journal & 95 \\
\hline Maita et al. & 2015 & & No & Journal & 0 \\
\hline $\begin{array}{c}\text { Breuker \& } \\
\text { Matzner }\end{array}$ & 2014 & & Systematised & Conference & 2 \\
\hline Yue et al. & 2011 & & No & Conference & 7 \\
\hline Qing-tian & 2007 & & No & Journal & 3 \\
\hline Wang et al. & 2011 & & No & Journal & 2 \\
\hline Yang \& Su & 2014 & \multirow{3}{*}{ Healthcare } & No & Conference & 1 \\
\hline Rojas et al. & 2015 & & No & Conference & 0 \\
\hline Rojas et al. & 2016 & & Yes & Journal & 0 \\
\hline
\end{tabular}

\section{Summary of Literature Reviews of Process Mining}

After reviewing the selected papers and clustering their main subjects, three main categories emerged: algorithms, tools, and healthcare.

\subsection{Algorithms}

In 2008, Tiwari et al. (2008) conducted a comprehensive literature review on process mining based on $50+$ research papers. They provided an overview of state-of-the-art techniques in order to identify current and future research trends, together with common problems. They also summarised the contribution of some soft computing techniques in process mining. The authors highlighted the application of genetic algorithms (de Medeiros et al., 2005), general algorithmic approaches (van der Aalst and Song, 2004), Markovian approaches (Cook and Wolf, 1998), neural networks (Schimm, 2004), and cluster analysis (Schimm, 2004) in process mining. In 44 of these papers, the share of general algorithmic approaches (other algorithms) in process mining is $62 \%$ (see Figure 9). The share of data mining-based approaches and soft computing techniques are only $15 \%$ and $9 \%$, respectively. In terms of notations, Petri nets were recognised as the most popular notation, with $29 \%$. 


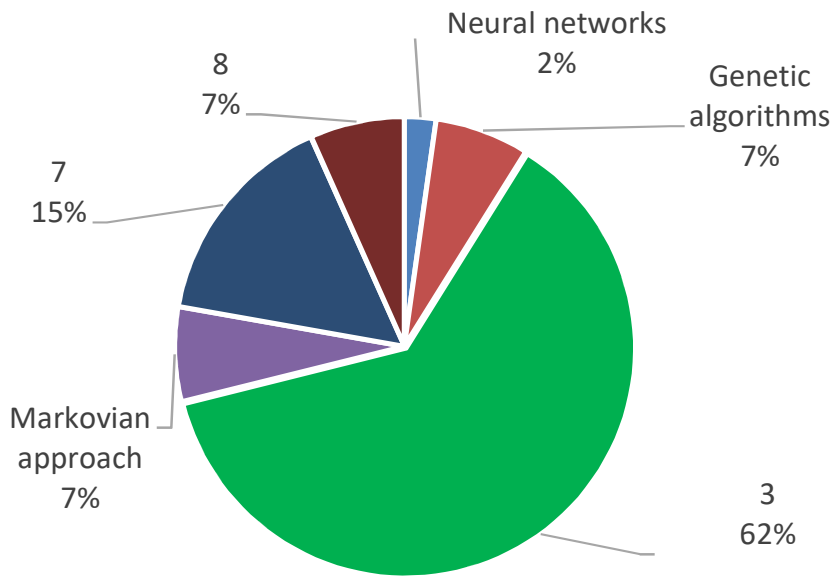

Figure 9. Share of Process Mining Techniques, as Reported by Tiwari et al. (2008)

Tiwari et al. (2008) also found that some of the papers attempted to address many processmining problems mentioned in Section 2. Noise in data is the most common problem reported in the papers $(20 \%)$ and noise mitigation techniques are widely used to tackle this problem. Mining loops and concurrent processes are also dealt with by several papers (17\% and $15 \%$, respectively). It is worth mentioning that some problems such as heterogeneous data sources and mining perspectives are not so well investigated, with only one paper each according to Tiwari et al. (2008). Although many process mining problems can be addressed using genetic algorithms, the most common problems (noise and mining loops) are still usually tackled with data mining-based approaches.

The concept of organizational routines was developed in management studies and organization sciences to assess collective frequent activity patterns. Breuker and Matzner (2014) focused on three kinds of process mining activities: discovery, conformance and enhancement. Breuker and Matzner reviewed the literature of both fields, i.e., process mining and organizational routines, to clarify their relation. Using Google Scholar and Scopus, they used a systematised approach to search for the papers from the field of organizational routines citing process mining, and they found nine papers. Six papers belonged to organizational routines but cited process mining (including three papers cited in both fields), while the remaining three cited both but did not belong to any of the fields. This review showed that researchers in these two fields employ different research methods and tools even though they work on similar research questions. This review concluded that using methods from the counter-field could create a great potential for research designs.

Yue et al. (2011) conducted a review on process mining algorithms. They introduced the work of some scholars and described their algorithms. To this end, the authors have categorised the contributors into Chinese and non-Chinese ones. In this review, 26 papers are studied, and the function of each algorithm is briefly introduced. The algorithms are mostly related to the process discovery topic.

Maita et al. (2015) focused their recent review on the use of artificial neural networks (ANNs) and support vector machines (SVMs) in process mining. Eleven papers were 
selected as primary studies. Their paper concludes that although process mining received significant research interest, less attention has been paid to ANNs and SVMs. This confirms the results of Tiwari et al. (2008) where only one paper had explored the neural network area in relation to process discovery (Cook and Wolf, 1998). The authors believe that this paucity exists mainly because researchers are generally not aware of these two data mining techniques and their potential for solving process mining problems.

Qing-tian (2007) studied the detailed definition, research approaches, algorithms, tools, and challenging research problems related with process mining. The author also reported on a subfield of process mining, distrusted process mining, together with its specific challenges.

Finally, Wang et al. (2014) considered discovery algorithms of process mining. The algorithms are compared, followed by a discussion of challenging research problems.

\subsection{Tools}

In this context, Claes and Poels (2013) conducted a survey about the opinion of people who used the ProM framework and its plugins. Despite the fact that this study is not a systematic literature review on process mining, it is worth considering as it provides a survey on the most prominent tool for process mining (ProM). Inspired by the Process Mining Manifesto, Claes and Poels created a questionnaire about process mining, ProM, and demographics. This survey was answered by 119 people from 26 different countries, with a bias from participants from The Netherlands and Belgium. The authors believe this is mainly caused by the process mining community having many members from these countries (van der Aalst et al., 2012). The authors captured the results of this exploratory survey as follows:

1. Benefits of process mining: objectivity, conformance checking.

2. Drawbacks of process mining: data access (to find and prepare the right data for process mining), too little guidance, hard-to-understand discovered models.

3. Popularity of process mining tools: the ProM framework, Disco.

The first result of this survey is aligned with the result of another survey by Ailenei et al. (2012) where the same question ("What do you think is the biggest benefit of process mining") was asked from participants.

Aligned with the second result of the survey from Claes and Poels (2013), Bose (2013) revealed that data quality is the main challenge in process mining research. Regarding the third result of the survey, several process mining techniques used by ProM were reported. The most frequently used plug-ins of ProM are Fuzzy Miner (Günther and van der Aalst), Heuristics Miner (Weijters et al., 2006), Social Network Miner (van der Aalst and Song, 2004), Dotted Chart Analysis (Song and van der Aalst, 2007) and the Alpha algorithm plugin (Li et al., 2007).

\subsection{Healthcare}

A clinical pathway clearly determines the goals and key elements of care (e.g., best practices and patient expectations), coordinating roles and sequencing the activities of the multidisciplinary care team. A clinical pathway aims to improve the quality of care, mitigate risks, increase patient satisfaction and increase the efficiency in the use of resources. Several research contributions have identified the lack of a uniformly accepted 
definition of a clinical pathway (Caron et al., 2013). Based on the literature review of De Bleser et al. (2006), there are 84 different terms that may be considered as synonyms of clinical pathway, including care map, care pathway, critical pathway, integrated care pathway, protocol and guideline.

Yang and Su (2014) focused on clinical pathways in a literature review that explains future directions in that area. This paper and the one from Rojas et al. (2015) are the only two reviews, resulting from our queries, related to process mining in healthcare. In Yang and Su's review, 37 papers published within 2004-2013 were selected and analysed. These papers are categorised in three areas: i) process discovery for clinical pathways design, ii) variants analysis and control, and iii) continuous evaluation and improvement.

In the first area, Yang and Su (2014) analysed the difficulties raised through applying process discovery techniques in healthcare processes, reported by 19 papers. The difficulties originated form the complex and unstructured nature of healthcare processes, noise, incompleteness, multiple occurrences of activities, and richness of process types and variants, which are some major challenges of mining clinical processes. Yang and Su have concluded that the diversity and complexity of medical processes in clinical pathways are far higher than that of common business processes. The medical environment also has a highly dynamic and uncertain nature, which adds to the complexity. Therefore, mining clinical pathway patterns, using traditional process mining techniques, faces many problems and challenges. Furthermore, the clinical pathways designed using the most recent approaches are non-adaptive and cannot adjust effectively when the external environment changes.

In the second area, 13 papers that mainly use conformance techniques and alignment were considered. In these papers, the variants of the clinical pathway are analysed to check the conformance of observed behaviour in the event logs with the model. Considering the techniques adopted in their selected papers, Yang and Su (2014) concluded that there are still many technical challenges for the identification and analysis of the clinical path variants. They believe that the identification and analysis methods are not always relevant and that they pay little attention to the sources and impacts of the variants, leading to low detection efficiency. The authors recommend that researchers analyse the variants from a systemic perspective, and consider the impacts on the adjustment of the clinical pathways.

In the third area, 11 papers related to continuous evaluation and improvement are discussed. They show that many researchers focus on the first two kinds of process mining (process discovery and conformance), but very limited studies have focused on the continuous evaluation and improvement of actual clinical pathways. According to Yang and $\mathrm{Su}$ (2014), the evaluation and improvement are regarded as the redesign of the clinical pathways. Nevertheless, the roots that triggered the improvement are always neglected. This neglect will rigorously impact the efficiency and effectiveness of continuous improvement. The authors emphasised that the continuous evaluation and improvement need to be supported by variants analysis. This analysis can find the root causes to ensure the effectiveness and pertinence of improvements.

Finally, the authors state four future research topics to meet the challenges of the medical environment. The further analysis of the variants is the basis for adaptive adjustment and improvement. Variants identification and analysis, customization of clinical pathways, self-learning improvement of clinical pathways, and integrated medical process management are the four key trends identified in the paper. 
Rojas et al. (2015) provided a bibliographic survey about process mining algorithms, tools, challenges, and case studies in healthcare processes. As this review was not done in a systematic fashion, the number of papers in the survey was not clearly mentioned. The publications cited in this paper and referring to at least one algorithm, tool, technique, or case study shows that 30 unique papers are covered by the survey. The paper presents an overview of the characteristics of different approaches including types of processes (three papers), types of data (two papers), process mining tools (eight papers), frequently posed questions (one paper), methods and algorithms (ten papers), used methodologies (three papers), and implementation strategies (seven papers). Moreover, the survey characterises some case studies (nine papers) according to their geographical location and their leading medical field.

Rojas et al. (2016) extended their previous paper (Rojas et al., 2015) and conducted another bibliographic survey about the application of process mining in healthcare covering 74 papers that report case studies in this context. Rojas et al. (2016) aim to provide a useful overview of the works undertaken in the field of process mining in healthcare and to help researchers to select process mining algorithms, tools, techniques, methodologies and approaches for their studies. To this end, they tried to identify and characterize the case studies, providing an overview of the state of the art of the field of process mining in healthcare. In addition, trends and challenges regarding the future of process mining in healthcare are discussed.

Rojas et al. (2016) have not sensitized or summarised the papers covered by their literature review; they have followed a systematic approach to search, find, include, and exclude papers. In contrast with Rojas et al. (2015), the methodology and the steps for selecting the papers for review are clearly described. They have looked for a combination of the keywords "process mining", "workflow mining" and "healthcare" using three search engines; PubMed, DBLP, and Google Scholar. In terms of inclusion and exclusion criteria, Rojas et al. (2016) included the articles that consist of a case study where process mining techniques or algorithms have been applied in the healthcare domain, published in English before February 2016. They excluded the papers that do not present a case study of process mining in healthcare. Moreover, the case studies concerning Clinical Pathways have been excluded. After removing duplications and considering the repository of the Health Analytics using Process Mining Team (Process Mining Group, 2014) from the Eindhoven University in the Netherlands, Rojas et al. (2016) finally selected 74 papers for their analysis: 22 papers from the web searches, 16 papers from the repository, and 36 from both places.

Aligned with what we found within our study, Rojas et al. (2016) reported that there is no comprehensive review of all case studies where process mining has been applied in the healthcare domain.

Rojas et al. (2016) did not aim to describe the contents of the papers they reviewed and consequently what has happened in the selected papers is not uncovered by their review. The approach characterizations from the two surveys of Rojas et al. $(2015,2016)$ are as follows:

- Types of process mining: In terms of types of process mining (Section 2.2), Rojas et al. (2015) discussed three papers about process discovery and two about conformance checking. Based on Rojas et al. (2016), 45 papers out of 74 (60\% of the case studies) have been related to process discovery concerning control flow and discovering the sequence of process activities. 
Conformance checking, which detects actual process deviations from predetermined model, has been used in 16 papers ( $21 \%$ of the case studies). The performance perspective, that allows improving the models based on analysing the execution time, identifying bottlenecks, idle time and synchronization time, has been applied in 10 papers (16\% of the case studies). Finally, the organizational perspective, which is based on analysing the collaboration between different resources, has been applied in nine of the 74 case studies (12\% of the case studies). Some papers have been considered in more than one of the aforementioned categories.

- Types of Processes: Rojas et al. (2015) observed that processes in the healthcare domain are categorised into two types. The medical treatment processes include activities from diagnostics to the execution of patient relief activities. The organizational processes focus on the organizational process, the collaborative information of the healthcare professionals and their organizational units. Three papers reviewed by Rojas et al. (2016) specify this classification of processes. However, another classification presented in one paper divided operational healthcare processes into two types: non-elective care, including medical emergencies; and elective care, which includes scheduled standard, routine and non-routine procedures.

- Types of Data: The types of data determine the processes capable of going through analysis. Also, the event logs are built using the content of these data. Rojas et al. (2016) found only two case studies representing classifications of data. The first one categorized the data into vital signs, events, and personal data of patients, whereas the second classification is established regarding the data source and its level of abstraction, accuracy, granularity, directness and correctness. This latter classification uses four data sources: administrative systems, clinical support systems, healthcare logistic systems, and medical devices. A clear understanding of the available data types supports researchers in building event logs and applying process mining in a correct manner (Rojas et al., 2016).

- Frequently Asked Questions: Mans and van der Aalst (2013) defined four typical questions to be answered by medical process specialists: i) What are the most followed paths and what exceptional paths are followed? ii) Are there any differences in care paths followed by different patient groups? iii) Do we comply with internal and external guidelines? iv) Where are the bottlenecks in the process? Rojas et al. (2015) proposed a fifth question in order to include the organizational collaboration process among specialists: What are the roles and social relationships between the medical staff? Two studies reviewed by Rojas et al. (2016) presented a classifications of frequently asked questions ( $3 \%$ of case studies).

- Techniques or Algorithms: Rojas et al. (2015) presented seven algorithms implemented to execute the desired analysis in process mining in healthcare. Most were discussed in the previous reviews: Trace Clustering (two papers), Fuzzy Miner (two papers), Alpha Miner (one paper), Genetic Miner (two papers), Heuristic Miner (three papers) and Conformance Checker (two papers). However, one algorithm was original: Performance Sequence Analyzer (two papers). Rojas et al. (2016) extracted 33 techniques or 
algorithms used in the case studies they reviewed and showed the number of cases that used each algorithm. The most commonly used techniques are Heuristics Miner (19 papers, 26\%), Fuzzy Miner (15 papers, 20\%), and Trace Clustering (Song et al., 2008) (8 paper, 11\%). This result is aligned with the result of Claes and Poels (2013) described in Section 5.2. As Rojas et al. (2016) showed, 19 algorithms that were extracted from 74 case studies are used only in one paper, so there is room for additional studies.

- Implementation Strategies: One original contribution of Rojas et al. (2015) is that they classified the strategies used to implement process mining in three classes based on their level of automation. The first one is the basic direct strategy, which utilises the process mining tools directly with an event log extracted directly from a data source (four papers). The second strategy is semi-automated, where a specific solution is needed to connect to one or several data sources and extract the data to build the event log (one paper). The third strategy is more automated. Here the steps are done in a specific suite, including connection to the data sources, extraction of the data, building of the event log and implementation of the process mining techniques. Here the person using the suites does not need knowledge of process mining tools in detail, but the suites are developed exclusively for an environment and its data sources. Medtrix Process Mining Studio (Ferreira, 2012) and the Emotiva Tool (Fernández-Llatas et al., 2013) are two example of such suites. In terms of the implementation strategies, Rojas et al. (2016) show that the first strategy (the direct one) is adhered to in 17 case studies $(23 \%)$, whereas the second strategy (the semi-automated one) is applied in only one case study. Finally, the third strategy (automated) is applied in 7 case studies (9\%).

- Process Mining Tools: In Section 2.5, we described the main tools available for process mining in general. Rojas et al. (2015) focused on the healthcare domain and considered seven papers to find the frequency of some main tools. They showed that ProM (van Dongen et al., 2005) has been used in a majority of case studies in healthcare. They, also, considered the other popular process mining tool Disco (Fluxicon, 2016), and showed that it is not mentioned as much as expected in the case studies that they reviewed. Aligned with these results, Rojas et al. (2016) concluded that ProM is the most common process mining tool used in healthcare, used in 31 of their case studies $(42 \%)$, followed by Disco ( 8 case studies, $11 \%$ ).

- Types of Case Studies: Rojas et al. (2015) defined three types based on the process mining tool they have used. The first type is the basic case study, in which there is no new implementation done and the objective is to provide knowledge of the healthcare process. A majority of the case studies have been related to of this type. In the second type, a new technique or algorithm has been developed as a complement to current tools. The third type is a mixture of tools and techniques used in cooperation with some techniques from other fields such as statistical analysis and data mining.

- Geographical Analysis of the Case Studies: Aligned with our results from Section 4.1, Rojas et al. (2015) show that use of process mining, as an important tool for analysing medical processes and generate improvements opportunities, has a growing trend in the recent years. Based on a quantitative 
count and geographical classification on the case studies available, the highest share of case studies is in Europe. There exist only a few in North America, Asia and Australia, and none in Africa or South America. Within the 74 case studies included by Rojas et al. (2016), around 73\% are in Europe, with a few examples in Australia, Asia and North America. Specifically, in Europe, The Netherlands is the country with the highest number of case studies, followed by Germany and Belgium.

- Medical Fields: Based on Rojas et al. (2015) in terms of the fields covered by the case studies, most have been done in oncology and surgery, and a few others in caregiving, cardiology, diabetes, dentistry, medication, intensive care, and radiotherapy. One year later, Rojas et al. (2016) identified 22 different fields where data were gathered. Oncology (9 case studies) and Surgery ( 8 case studies) are again the medical fields with the highest numbers of case studies. Analysing the distribution of case studies within 22 medical fields shows the multidisciplinary character of process mining in healthcare, together with its potential application to all medical fields (Rojas et al., 2016).

- Analysis Strategies: Rojas et al. (2016) established the use of three analysis strategies from the literature review and the case studies identified. These strategies are based on the way in which the task of applying process mining techniques and algorithms is undertaken. In the first strategy, namely basic strategy, the process mining techniques and algorithms prevalent in the available tools are applied. This is the easiest strategy to perform without using any new technique or algorithm, or techniques from other areas (15 papers, $19 \%$ of the cases reviewed). In the second strategy, a new process mining technique or algorithm with an objective particular to each specific case study is implemented. The main objective with the new implementation is to discover novel ways to deal with processes that are flexible, unstructured and complex, and also to handle large, multidimensional datasets (6 case studies, 8\%). In the third and last strategy, in addition to current techniques and algorithms in current tools, researcher incorporates analysis of other areas, such as statistical analysis (10 papers, 13\%), data mining (4 papers, 5\%), ontologies ( 2 papers, $3 \%$ ) or simulation models ( 1 paper, $1 \%$ ). It is noteworthy that the resource consumption, the complexity and the required expertise in the second strategy are higher than for the others. Yet, the third strategy is also challenging because forming a team of analysts using the techniques from several areas is required (Rojas et al., 2016).

- Challenges: The limitations identified by Rojas et al. (2015) relate to data (seven challenges) and to the involvement of expert medical knowledge (two challenges). Aligned with the results of Tiwari et al. (2008) in Section 5.1, the limitations related to data are the main challenges of process mining even in the healthcare domain: identifying and accessing data sources; including the physical information and conditions of the patients; data integration from different sources; data quality; granularity and pre-processing of the data; using real event logs and data; and building a correct and complete event log. The second category of challenges includes satisfying medical protocols and guidelines, and including medical knowledge. According to Rojas et al. (2016), one of the weaknesses of current process mining tools is the absence of a good visualization of the process models, especially for complex and less- 
structured processes such as those in the healthcare domain. A great amount of reliance on experts for applying process mining is another challenge. The tools or solutions that are straightforward to apply, without the need for deep knowledge of the tools and techniques, are yet to be developed.

The results of these three healthcare-oriented reviews are hence in line with the results from the general process mining reviews, with additional challenges that require further effort and attention.

\section{Threats to Validity}

The goal of a systematic review is to uncover as many primary relevant studies as possible to summarise all current information about some phenomenon in a systematic and unbiased manner. There are several threats to the validity of our research and of the papers that were reviewed in Section 5. Validity of a research is concerned with the question about the correctness of conclusions, i.e. the alignment between conclusions and reality (Pitman, 1998).

In this section, we consider the threats and the biases that could affect the validity of our work and of the eleven papers selected for review. The main threats are discussed according to two categories: construct validity and internal validity (Feldt and Magazinius, 2010).

\subsection{Construct Validity}

Construct validity refers to the quality of the methodology in terms of being helpful to answer the target research questions. In our paper, searching for and selecting relevant papers played an essential role. Note that our first question (RQ1) was directly answered based on counting the papers that are related to process mining, while the two other questions (RQ2 and RQ3) were answered based on some papers selected from the set found during the searching stage. Therefore, any weakness and inaccuracy in the first stage could threaten the correctness of our answers. Also, regarding the papers we reviewed, all but three papers (Qing-tian, 2007; Wang et al., 2011; de Medeiros et al., 2005) have clearly used search engines to provide their paper sets. As these papers are literature reviews, their research questions are answered based on the results of their queries. Therefore, their validity level is depended on their choice of queries, databases, and search engines. Unfortunately, only Breuker and Matzner (2015) have reported the steps of their search, including search engines (Google Scholar and Scopus), keywords and inclusion and exclusion criteria. Here, two categories of threats that are associated with selecting papers are discussed.

Missing some relevant references: Although we included the most frequent and essential keywords in the queries, there is a possibility that some relevant papers have not been found. For example, the very first paper in the context of process mining (Cook, 1996), was not found by our queries, as it focused on "process discovery" rather than on process mining. Breuker and Matzner (2015) also used "process mining" in their query.

The second threat is related to the search engine limitations. As shown in Figure 5, 46\% of our collected papers are returned only by Google Scholar. This indicates that this search engine is a significant contributor of relevant references. On the other hand, as Google 
Scholar only gives the possibility of search within title or full-text, we opted to search in titles only (to avoid noise). Consequently, the papers that do not have the queries' keywords in their titles were not detected by Google Scholar.

Given that we have defined our queries' keywords in English, another threat that may affect the validity of our review (and the eleven papers we reviewed) is the likelihood of ignoring some relevant non-English papers. To our knowledge, only Yue et al. (2011) considered some non-English papers (in Chinese).

Finally, taking advantage of four search engines, including two major ones (Google Scholar and Scopus), enables us and Breuker and Matzner (2015) to detect a large proportion of the papers stored in scientific databases. However, there might be some relevant papers stored in other databases that are not accessible with these search engines.

Collecting irrelevant references: The threats in this category are related to finding irrelevant papers through the queries. As described in section 4.1, we iteratively used more constraints to filter out some irrelevant papers, specifically the ones related to the mining or chemical industry. Yet, reviewing every single paper is the only way to make sure that there is no remaining irrelevant paper. We performed this approach on the 36 selected papers. Breuker and Matzner (2015) have also used some automatic filtering to exclude some papers. They have found 2016 papers for process mining and 5693 for organizational routines. Obviously, these high numbers suggest the papers were not reviewed manually.

One of the important parts of a systematic literature review is to clarify the study selection criteria. These criteria are intended to identify the relevant studies that convey direct evidence related to the research question while avoiding bias (Yue et al., 2011). Regarding the papers considered in Section 5, as seen from Table 5, only two (Breuker and Matzner, 2015; Rojas et al., 2016) have clarified their selection criteria.

According to these two kinds of threats, there is a chance that our trend-graphs may not be precise (RQ1). Also, the selected papers may not capture all papers that are published related to literature reviews in process mining (RQ2 and RQ3).

\subsection{Internal Validity}

Some other biases and confusing factors can threaten validity of our study and the studies that we reviewed. For example, Maita et al. (2015) focused on the use of artificial neural networks and support vector machines in process mining. They reviewed 11 papers and concluded that the attention that has been paid to ANNs and SVMs by process mining researchers is less than expected. Then, they related this to process mining researchers' ignorance of these two data mining techniques. These two conclusions could be wrong because of the bias of the authors.

In this paper, one additional threat is that the selection of papers and the application of the criteria was mainly done by one person (the first author) in a relatively short time. We suspect the other eleven papers to be in a similar situation (but such threats are rarely discussed in their papers). Such issue could be mitigated by having more people do such selection independently, and then with a consensus mechanism.

\section{Conclusions}

This paper first provided an overview of process mining in general, and then an example of application to the healthcare domain in particular. Following the systematised literature 
review approach, we ran queries through six relevant search engines and analysed the returned papers to answer three research questions (see Section 4).

Regarding question RQ1, we conclude that process mining is a growing research topic, which emerged in the last decade. Also, we observed that the number of publications related to process mining in healthcare is also increasing, at an even higher rate.

Question RQ2 was about the existence of systematic literature reviews specifically related to process mining and, also, care processes. We obtained, categorised and summarised eleven such review papers. We found only three literature reviews about process mining in healthcare. The first one focused on clinical pathways and the second and the third ones provided a bibliographic survey about the process mining algorithms, tools, case studies and challenges in the analysis of healthcare processes without synthesising or summarising the papers they reviewed.

The last question (RQ3) was about the quality of the existing literature reviews in terms of giving a good understanding about process mining, especially in healthcare. The review of the papers showed that although there exist some literature reviews on process mining, they do not provide a comprehensive and up to date view of this area. The review, also, showed that only one of these literature reviews is conducted in a systematic fashion, and threats to validity are rarely discussed explicitly.

Although this systematised literature survey provides clear indications of trends and a good overview of the status of process mining in healthcare, it does so mainly through the lens of other literature reviews. For future work, it is recommended to conduct a systematic literature review (Kitchenham et al., 2009) of process mining, especially in the healthcare domain, with non-review papers as first-class entities (e.g., using the 168 papers mentioned in Figure 8, and newer papers). Studies on the cost-effectiveness of process mining in healthcare would also be of high interest.

\section{References}

All URLs were last accessed in March 2016.

Agarwal, N. and Singh, L. (2014) 'Process Mining Tools: A comparative Analysis and Review', Advances in Computer Science and Information Technology (ACSIT), 1(2), pp.26-29.

Ailenei, I., Rozinat, A., Eckert, A., and van der Aalst, W.M.P. (2012) 'Definition and validation of process mining use cases', Business Process Management Workshops, LNBIP 99, Springer, pp.75-86.

Aldin, L. and de Cesare, S. (2011) 'A literature review on business process modelling: new frontiers of reusability', Enterp. Inf. Syst., 5(3), pp.359-383.

Bergenthum, R., Desel, J., Lorenz, R., and Mauser, S. (2007) 'Process mining based on regions of languages', Business Process Management, LNCS 4714, Springer, pp.375-383.

Bose, R.P.J.C. (2013) 'Wanna Improve Process Mining Results? It's High Time We Consider Data Quality Issues Seriously', IEEE Symp. on Computational Intelligence and Data Mining (CIDM 2013), IEEE CS, pp. 127-134.

Breuker, D. and Matzner, M. (2014) 'Performances of business processes and organizational routines: Similar research problems, different research methods - A literature review', Proceedings of the European Conference on Information Systems (ECIS 2014), AISeL, pp.113. [online] http://aisel.aisnet.org/cgi/viewcontent.cgi?article=1242\&context=ecis2014

Caron, F., Vanthienen, J., Vanhaecht, K., Van Limbergen, E., Deweerdt, J., and Baesens, B. (2013) 'A process mining-based investigation of adverse events in care processes', Health Information Management Journal, 43(1), pp.16-25.

Celonis (2016) Celonis Process Mining. [online] http://www.celonis.de/en/ 
Claes, J. and Poels, G. (2013) 'Process mining and the ProM framework: an exploratory survey', Business Process Management Workshops, LNBIP 132, Springer, pp.187-198.

Cook, J.E. (1996) Process discovery and validation through event-data analysis, Ph.D. thesis, Dept. Computer Science, University of Colorado, USA. Technical report CI-CS-817-96.

Cook, J.E. and Wolf, A.L. (1998) 'Discovering models of software processes from event-based data', ACM Trans. Softw. Eng. Methodol., 7(3), pp.215-249.

De Bleser, L., Depreitere, R., De Waele, K., Vanhaecht, K. , Vlayen, J., and Sermeus, W. (2006) 'Defining pathways', J. Nurs. Manag., 14(7), pp.553-563.

de Medeiros, A.K.A., Weijters, A.J.M.M., and van der Aalst, W.M.P., (2005) Using genetic algorithms to mine process models: representation, operators and results, BETA Working Paper Series, WP 124, Eindhoven University of Technology, The Netherlands.

de Medeiros, A.K.A., Weijters, A.J.M.M., van der Aalst, W.M.P., and de Medeiros, A. (2007) 'Genetic process mining: an experimental evaluation', Data Min. Knowl. Discov., 14(2), pp.245-304.

de Medeiros, A.K.A., van der Aalst, W.M.P., Karla, A. and Pedrinaci, C. (2008) 'Semantic process mining tools: core building blocks', ECIS 2008 Proceedings, paper 96, AISeL. [online] http://aisel.aisnet.org/ecis2008/96

Feldt, R. and Magazinius, A. (2010) 'Validity Threats in Empirical Software Engineering ResearchAn Initial Survey', 22nd Int. Conf. on Software Engineering \& Knowledge Engineering (SEKE'2010), Knowledge Systems Institute Graduate School, pp.374-379.

Fernández-Llatas, C., Benedi, J.-M., Garcia-Gómez, J.M., and Traver, V. (2013) 'Process Mining for Individualized Behavior Modeling Using Wireless Tracking in Nursing Homes', Sensors, 13(11), pp.15434-15451.

Ferreira, D.R. (2012) 'Business process analysis in healthcare environments: A methodology based on process mining', Information Systems, 37(2), pp.99-116.

Fluxicon (2016) Disco. [online] https://fluxicon.com/disco/

Fujitsu (2016) Automated Process Discovery (APD). [online] http://www.fujitsu.com/global/ products/software/middleware/application-infrastructure/interstage/solutions/bpmgt/bpm/

Günther, C.W. and van der Aalst, W.M.P. (2007) 'Fuzzy Mining - Adaptive Process Simplification Based on Multi-perspective Metrics', Business Process Management, LNCS 4714, Springer, pp.328-343.

ISO/IEC (2004) ISO/IEC 15909-1:2004. Systems and software engineering - High-level Petri nets Part 1: Concepts, definitions and graphical notation.

Kitchenham, B., Brereton, O.P., Budgen, D., Turner, M. Bailey, J., and Linkman S. (2009) 'Systematic literature reviews in software engineering - A systematic literature review', Inf. Softw. Technol., 51(1), pp.7-15.

Lexmark International (2016) Perceptive Process Mining. [online] http://www.lexmark.com/en_us/ products/software/workflow-and-case-management/process-mining.html

Li, J., Liu, D., and Yang, B. (2007) 'Process Mining: Extending $\alpha$-Algorithm to Mine Duplicate Tasks in Process Logs', Advances in Web and Network Technologies, and Information Management, LNCS 4537, Springer, pp.396-407.

Maita, A.R.C., Martins, L.C., López Paz, C.R., Peres, S.M., and Fantinato, M. (2015) 'Process mining through artificial neural networks and support vector machines', Bus. Process Manag. J., 21(6), pp.1391-1415.

Mans, R., van der Aalst, W.M.P., and Vanwersch, R. (2015) Process Mining in Healthcare Evaluating and Exploiting Operational Healthcare Processes. SpringerBriefs in Business Process Management, Springer International Publishing, 2015.

Mans, R., van der Aalst, W.M.P., Vanwersch, R., and Moleman, A.J. (2013) 'Process Mining in Healthcare: Data Challenges When Answering Frequently Posed Questions', Process Support and Knowledge Representation in Health Care, LNCS 7738, Springer, pp.140-153.

OMG (2011) Business Process Model and Notation (BPMN) Version 2.0, formal/2011-01-03.

Pitman, M. (1998) 'Qualitative Research Design: An Interactive Approach', Anthropol. Educ. Q., 29(4), pp.499-501.

Process Mining Group (2014) Papers About Process Mining in Healthcare [online] http://www.processmining.org/health/papers

Process Mining Group (2010) ProM Tools. [online] http://www.promtools.org/doku.php 
Qing-tian, Z. (2007) 'A Survey of Research Issues and Approaches on Process Mining', J. Syst. Simul., 2007-S1.

QPR (2015) QPR ProcessAnalyzer. [online] http://www.qpr.com/products/qpr-processanalyzer

Rojas, E., Arias, M., and Sepúlveda, M. (2015) 'Clinical processes and its data, what can we do with them?', 8th International Conference on Health Informatics (HEALTHINF-2015), SCITEPRESS, pp.642-647.

Rojas, E., Munoz-Gama, J., Sepúlveda, M., and Capurro, D., (2016) 'Process Mining in Healthcare: A literature review', Journal of Biomedical Informatics, 61, pp.224-236.

Rozinat A. and van der Aalst, W.M.P. (2008) 'Conformance checking of processes based on monitoring real behavior', Inf. Syst., 33(1), pp.64-95.

Rozinat, A., de Medeiros, AK.A., Günther, C.W., Weijters, A.J.M.M., and van der Aalst, W.M.P. (2007) Towards an evaluation framework for process mining algorithms. BPM report 0706, BPMcenter.org, [online] https://pure.tue.nl/ws/files/2263202/731400825770131.pdf

Schimm, G. (2004) 'Mining exact models of concurrent workflows', Comput. Ind., 53(3), pp.265281.

Software AG (2012) ARIS Process Performance Manager. [online] http://www.softwareag.com/nl/ products/aris_platform/aris_controlling/aris_process_performance/overview/default.asp

Song, M. and van der Aalst, W.M.P. (2007) 'Supporting process mining by showing events at a glance', 17th Annual Workshop on Information Technologies and Systems (WITS 2007), pp.139-147.

Song, M., Günther, C.W., and Van der Aalst, W.M., (2008) 'Trace clustering in process mining'. Business Process Management Workshops, Springer, pp.109-120.

The World Bank (2015) Health expenditure, total (\% of GDP). [online] http://data.worldbank.org/ indicator/SH.XPD.TOTL.ZS/countries/1W-CA-US-EU-CN-ZA-GB?display=graph

Tiwari, A., Turner, C.J., and Majeed, B. (2008) 'A review of business process mining: state-of-theart and future trends', Bus. Process Manag. J., 14(1), pp.5-22.

van der Aalst, W.M.P. (2011) Process mining: discovery, conformance and enhancement of business processes. Springer Science \& Business Media, 2011.

van der Aalst, W.M.P. (2012) 'What makes a good process model?', Softw. Syst. Model., 11(4), pp.557-569.

van der Aalst, W.M.P. and Song, M. (2004) 'Mining Social Networks: Uncovering Interaction Patterns in Business Processes', International Conference on Business Process Management (BPM 2004), LNCS 3080, Springer, pp. 244-260, 2004.

van der Aalst, W.M.P. and Weijters, A.J.M.M. (2004) 'Process mining: a research agenda', Comput. Ind., 53(3), pp.231-244.

van der Aalst, W.M.P., Weijters, T., and Maruster, L. (2004) 'Workflow mining: Discovering process models from event logs', IEEE Trans. Knowl. Data Eng., 16(9), pp.1128-1142.

van der Aalst, W.M.P. et al. (2012) 'Process mining manifesto', Business Process Management Workshops, LNBIP 99, Springer, pp.169-194.

van Dongen, B., de Medeiros, A.K.A., Verbeek, H.M.W., Weijters, A.J.M.M., and van der Aalst, W.M.P. (2005) 'The ProM framework: A new era in process mining tool support', Applications and Theory of Petri Nets 2005, LNCS 3536, Springer, pp.444-454.

Wang, J., Tang, D., and Xie, Y. (2011) 'A Survey of Research on Process Mining Algorithms', Fire Control Command Control, Issue 8, pp.5-10.

Wang, J., Wong, R.K., Ding, J., Guo, Q., and Wen, L. (2013) 'Efficient Selection of Process Mining Algorithms', IEEE Trans. Serv. Comput., 6(4), pp.484-496.

Weijters, A.J.M.M. and Ribeiro, J.J.T.S. (2011) 'Flexible Heuristics Miner (FHM)', 2011 IEEE Symposium on Computational Intelligence and Data Mining (CIDM), IEEE CS, pp.310-317.

Weijters, A.J.M.M., van der Aalst, W.M.P., and de Medeiros, A.K.A. (2006) Process Mining with the HeuristicsMiner Algorithm, BETA Working Paper Series, WP 166, Eindhoven University of Technology, The Netherlands.

XES (2016) Extensible Event Stream-XES. [online] http://www.xes-standard.org/

Yang, W. and Su, Q. (2014) 'Process mining for clinical pathway: Literature review and future directions', 11th International Conference on Service Systems and Service Management (ICSSSM), IEEE CS, pp. 1-5. 
Yue, D., Wu, X., Wang, H., and Bai, J. (2011) 'A review of process mining algorithms', 2011 International Conference on Business Management and Electronic Information (BMEI), vol. 5, IEEE, pp.181-185. 